\title{
Combined influence of beamstrahlung and coupling impedance on beam energy spread and length in future lepton colliders
}

\author{
D. Leshenok $\odot^{*}$ and S. Nikitin $\odot^{\dagger}$ \\ Budker Institute of Nuclear Physics, 630090 Novosibirsk, Russia \\ Y. Zhang $\oplus^{*}$ \\ IHEP, 19B Yuquan Road, Shijingshan District, Beijing 100049, China \\ M. Zobov ${ }^{\S}$ \\ INFN, Laboratori Nazionali di Frascati, via Enrico Fermi 40, 00044 Frascati, RM, Italy
}

(Received 20 July 2020; accepted 30 September 2020; published 27 October 2020)

\begin{abstract}
A semianalytical model has been developed to study a combined effect of beamstrahlung due to beambeam interaction and beam coupling impedance in the future lepton colliders CEPC and FCC-ee. This model allows evaluating an impact of the coupling impedance on the bunch length and energy spread in collision. The model is benchmarked against numerical simulations. Analytical estimates for the supercolliders are presented.
\end{abstract}

DOI: 10.1103/PhysRevAccelBeams.23.101003

\section{INTRODUCTION}

To perform very high precision physics experiments, the future supercolliders FCC-ee [1] and CEPC [2] are going to exploit the crab-waist (CW) collision scheme [3,4] to push their luminosity. The beamstrahlung (BS) effect leading to a substantial bunch lengthening and energy spread increase becomes important [5] due to the tiny sizes of intense beams to be used in collision. In the crab-waist collisions with a large Piwinski angle, the bunch length increase results in the luminosity reduction, while the energy spread affects the experiment energy resolution. In turn, the beam coupling impedance is also responsible for the bunch lengthening, and, in addition, it can result in the energy spread increase if the microwave instability threshold is exceeded. Moreover, the imaginary part of the impedance reduces the synchrotron frequency due to the potential well distortion effect (PWD, see, for example, [6]), and the collision working point is to be reoptimized to compensate for this shift.

The interplay of both effects can change substantially the final values of the bunch length, the energy spread, and the

\footnotetext{
*dariya210612@gmail.com

nikitins@inp.nsk.su

zhangy@ihep.ac.cn

${ }^{\S}$ Mikhail.Zobov@lnf.infn.it
}

Published by the American Physical Society under the terms of the Creative Commons Attribution 4.0 International license. Further distribution of this work must maintain attribution to the author(s) and the published article's title, journal citation, and DOI. synchrotron tune. For example, the energy spread due to BS can suppress the microwave instability, and a longer bunch will produce a weaker wake potential. On the other hand, the impedance-related bunch lengthening is expected to reduce the strength of the $\mathrm{BS}$ effect.

The 3D self-consistent numerical simulations of the combined effect of beam-beam interaction and the beam coupling impedance require a huge computational time [7].

In this paper, we present a semianalytical model (first described in Ref. [8]) that allows evaluating the bunch length, energy spread, and synchrotron frequency in a much shorter time. The typical calculation time on a standard notebook is about several minutes. For comparison, to complete the simulations including the impedance presented in Fig. 2, it took about $16 \mathrm{~h}$ of CPU time using 36 CPU cores. The results obtained for FCC-ee and CEPC by using the model are compared with available numerical simulation data.

\section{ENERGY LOSS DUE TO BS}

The CW collision scheme proposed and tested at Frascati has helped to increase luminosity in the operating colliders DAФNE [4] and SuperKEKB [9], and now it is considered for application in all future lepton collider projects such as FCC-ee [1], CEPC [2], Super Charm-Tau factories SCT [10] in Russia, and HIEPA [11] in China.

This collision scheme makes it possible to focus highintensity bunches on a very tiny collision spot and, at the same time, allows overcoming the problem of their electromagnetic interaction by suppressing nonlinear resonances excited in beam-beam collisions. This is achieved by 


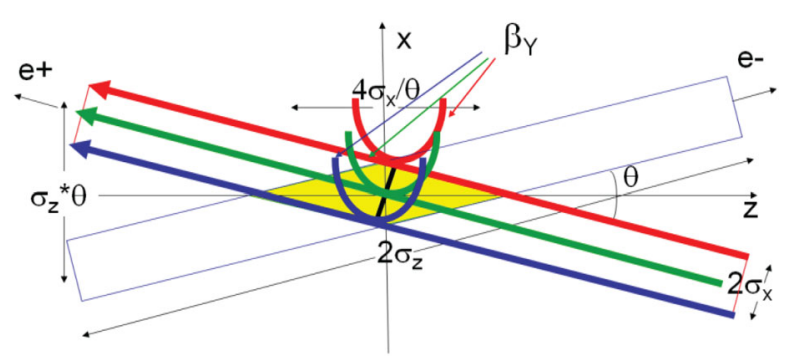

FIG. 1. Collision with a large Piwinski angle.

exploiting a very large Piwinski angle in collisions, providing low betatron functions at the interaction point, and by using dedicated sextupoles installed in the interaction region [3].

Let the electron and positron bunches colliding at the crossing angle $\theta \ll 1$ contain $N$ particles each, and $\boldsymbol{\sigma}_{x}, \boldsymbol{\sigma}_{y}$, and $\boldsymbol{\sigma}_{z}$ are their horizontal, vertical, and longitudinal sizes, respectively (see Fig. 1).

The Piwinski angle relates the geometric collision angle and the ratio of the longitudinal and transverse beam sizes $\Psi=\frac{\sigma_{z}}{\sigma_{x}} \tan \frac{\theta}{2}$. This characteristic parameter enters in the luminosity formula, and it is also a measure of the beambeam synchrobetatron resonance strength [12]. As is seen in Fig. 1, for a large Piwinski angle the collision area becomes small, and the beams can be focused on that small area without incurring the hourglass effect [13]. In that case, the vertical beta function $\beta_{y}$ can be made much smaller than the bunch length $\sigma_{z}$. Finally, the dedicated sextupoles help to eliminate the beam-beam resonances arising (in collision without $\mathrm{CW}$ ) due to the vertical motion modulation by the horizontal betatron oscillations [14].

For the future high energy colliders FCC-ee and CEPC, the beam sizes get so small that the BS becomes important for the collider performance.

In order to estimate the average particle energy loss due to radiation in the collective field of the oncoming bunch (BS), we use the approach developed by one of the authors in Refs. $[15,16]$. It is based on using the Takayama potential [17] of a 3D Gaussian charge distribution. The BS energy losses are calculated in the rest frame of the oncoming bunch and then recounted in the laboratory system. For the isolated case of a head-on collision, loss averaging is performed over all particles of the test bunch. For a nonzero but small crossing angle, averaging is limited, for simplification, by the region of the central cross section of the bunch. In the latter case, the ratio of the average loss to the initial particle energy is found from the equation $\left(\boldsymbol{\sigma}_{z} \gg \boldsymbol{\sigma}_{x}, \boldsymbol{\sigma}_{y}\right)$ [16]

$$
\begin{aligned}
U_{B S}= & \left\langle\frac{\Delta E_{B S}}{E}\right\rangle \approx \frac{4}{3} \frac{r_{e}^{3} N^{2} \gamma}{\sqrt{\pi} \sigma_{z}} \iint_{0}^{\infty} \frac{d t d t^{\prime}}{\Omega \sqrt{\left(2 \sigma_{x}^{2}+t\right)\left(2 \sigma_{x}^{2}+t^{\prime}\right)}} \\
& \cdot\left[\frac{\sigma_{x}^{2}}{g_{x} \sqrt{g_{y}}}\left(1+\frac{\theta^{2} \sigma_{z}^{2}}{8 \sigma_{x}^{2} \Omega^{2}}\right)+\frac{\sigma_{y}^{2}}{g_{y}^{3 / 2}}\right] .
\end{aligned}
$$

Here $r_{e}$ is the classical electron radius; $\gamma$ the relativistic factor of beam particles;

$$
\begin{aligned}
& g_{x}\left(t, t^{\prime}\right)=\left(2 \sigma_{x}^{2}+t\right)\left(2 \sigma_{x}^{2}+t^{\prime}\right)+2 \sigma_{x}^{2}\left(4 \sigma_{x}^{2}+t+t^{\prime}\right), \\
& g_{y}\left(t, t^{\prime}\right)=\left(2 \sigma_{y}^{2}+t\right)\left(2 \sigma_{y}^{2}+t^{\prime}\right)+2 \sigma_{y}^{2}\left(4 \sigma_{y}^{2}+t+t^{\prime}\right) ;
\end{aligned}
$$

the functional

$$
\Omega=\sqrt{1+\left[\frac{1}{2 \sigma_{x}^{2}+t}+\frac{1}{2 \sigma_{x}^{2}+t^{\prime}}\right] \frac{\sigma_{z}^{2} \theta^{2}}{4}}
$$

represents a generalized Piwinski factor. When $t=t^{\prime}=0$, $\Omega$ takes the known form

$$
\Omega(0,0)=\sqrt{1+\left(\frac{\sigma_{z} \theta}{2 \sigma_{x}}\right)^{2}}=\sqrt{1+\Psi^{2}} .
$$

The numerical evaluation of the double integral in Eq. (1) takes less than a minute on a conventional PC. The formula (1) allows estimating the influence of BS on the formation of the longitudinal beam size and energy spread in supercolliders [18]. To this aim, the approximation of the interaction region in the form of an equivalent magnet with some effective values of the field and length can serve as the simplest model. In the theory of synchrotron radiation, losses in such a magnet are proportional to the product of the square of its field by the length. At large Piwinski "angle" $\Psi \gg 1$ as takes place in the crab-waist interaction region of the supercolliders, the size $L_{\text {eff }}=$ $4 \sigma_{x} / \theta$ (see Fig. 1) can be approximately considered as a full effective length of the "magnet."

The model assumes that the beams retain their shape and sizes. In reality, there is an increase of the transverse bunch sizes at distances comparable with or larger than $\beta_{y}^{*}\left(\beta_{x}^{*}\right)$, the vertical (horizontal) beta function value at the interaction point (IP) (hourglass effect). This occurs vertically, starting from rather short distances, in comparison with the horizontal direction, since the vertical beta function is much smaller than the horizontal one. To verify this effect, the growth rate of losses was calculated depending on the distance of the center of the test bunch to the IP with and without taking into account the corresponding change in vertical size at the FCC-ee example [16]. This numerical experiment demonstrated the almost complete insensitivity of the model to the hourglass, which can be explained by the hierarchy of bunch sizes and the fact that the beams intersect in the median plane.

\section{BEAM ENERGY SPREAD AND LENGTH DUE TO BS}

To determine the steady-state beam energy spread and a self-consistent estimate of the length $\sigma_{z}$ as a result of $\mathrm{BS}$, 
we use the equation of radiative diffusion of the particle energy taking into account radiative damping [19]:

$$
\frac{d\left\langle A^{2}\right\rangle}{d t}=D_{\gamma}-2 \frac{\left\langle A^{2}\right\rangle}{\tau_{E}} .
$$

Here $\left\langle A^{2}\right\rangle$ is the average square of the energy oscillation amplitude in a beam; $\tau_{E}$ is the corresponding damping time; $D_{\gamma}$ is a sum of the coefficients of diffusion due to synchrotron radiation (SR) in the bending magnets and BS in the IP: $D_{\gamma}=D_{B S}+D_{S R}$. The $D_{S R}$ value was taken based on the design data of the collider magnetic structure. $D_{B S}$ was estimated by the formula

$$
D_{B S}=n_{I P} f_{0} C_{u} u_{c} P_{\gamma} \frac{L_{\mathrm{eff}}}{c}
$$

with $n_{I P}$ the number of interaction points which are considered identical to each other (in our case, $n_{I P}=2$ ); $f_{0}$ the revolution frequency; $C_{u}=1.32 ; u_{c}=3 \hbar c \gamma^{3} /(2 \rho)$ the characteristic radiation quantum in the "long magnet" approximation; $\hbar$ the Planck constant; $P_{\gamma}=c E U_{B S} / L_{\text {eff }}$ the power defined through the energy loss for BS (4); $L_{\text {eff }}$ the "equivalent magnet" length determined in the section above; $\rho=\sqrt{2 \pi P_{\gamma} /\left(c C_{\gamma} E^{4}\right)}$ the curvature radius of particle trajectory in the "equivalent magnet"; and $C_{\gamma}=8.85 \times 10^{-5}$ meter $\mathrm{GeV}^{-3}$. In a study of the BS spectrum, it was shown [20] that the approximation of a "long" (ordinary) magnet still remains relevant as applied to the present projects of supercolliders. It does not need to be changed to the "short magnet" model.

Since $U_{B S}$ in Eq. (1) depends on the beam length $\sigma_{z}$, and that in turn it is proportional to the energy spread $\sigma_{E}$, the stationary solution (3) is found from the transcendental equation

$$
\sigma_{E}^{2}=\frac{1}{4 E^{2}} \tau_{E} D_{\gamma}\left(\sigma_{z}\right)
$$

with

$$
\sigma_{z}=\frac{c \alpha_{p}}{\nu_{s} \omega_{0}} \cdot \sigma_{E}
$$

$\alpha_{p}$ is the momentum compaction factor, $\nu_{s}$ is the synchrotron tune, and $\omega_{0}=2 \pi f_{0}$. The system of Eqs. (4)-(6) is solved iteratively, which gives self-consistent values of the beam length and energy spread taking BS into account. It is assumed that the beam transverse dimensions at the IP remain constant. This is true, first, because with the nominal beam parameters the beam-beam limit is not reached. Second, due to zero dispersion in the interaction region, BS does not lead to an additional increase in the transverse emittances.
TABLE I. The bunch energy spread and length due to the $\mathrm{SR}+\mathrm{BS}$ effect at CEPC.

\begin{tabular}{lccc}
\hline \hline$E[\mathrm{GeV}]$ & 45.5 & 80 & 120 \\
$\sigma_{E}$ & $0.00110^{\mathrm{a}}$ & $\cdots$ & $0.00146^{\mathrm{a}}$ \\
& $0.00108^{\mathrm{b}}$ & $0.00111^{\mathrm{b}}$ & $0.00137^{\mathrm{b}}$ \\
$\sigma_{z}[\mathrm{~mm}]$ & $7.0^{\mathrm{a}}$ & $\cdots$ & $4.0^{\mathrm{a}}$ \\
& $6.8^{\mathrm{b}}$ & $4.9^{\mathrm{b}}$ & $3.7^{\mathrm{b}}$ \\
\hline \hline
\end{tabular}

${ }^{\mathrm{a}}$ Beam-beam simulation [21].

${ }^{\mathrm{b}}$ Semianalytical model (SR + BS).

TABLE II. The bunch energy spread and length due to the $\mathrm{SR}+\mathrm{BS}$ effect at FCC-ee.

\begin{tabular}{lccc}
\hline \hline$E[\mathrm{GeV}]$ & 45.6 & 80 & 120 \\
$\sigma_{E}$ & $0.00132^{\mathrm{a}}$ & $0.00131^{\mathrm{a}}$ & $0.00165^{\mathrm{a}}$ \\
& $0.00135^{\mathrm{b}}$ & $0.00130^{\mathrm{b}}$ & $0.00166^{\mathrm{b}}$ \\
$\sigma_{z}[\mathrm{~mm}]$ & $12.1^{\mathrm{a}}$ & $6.0^{\mathrm{a}}$ & $5.3^{\mathrm{a}}$ \\
& $12.4^{\mathrm{b}}$ & $5.9^{\mathrm{b}}$ & $5.3^{\mathrm{b}}$ \\
\hline \hline
\end{tabular}

${ }^{\mathrm{a}}$ Beam-beam simulation $[1,23]$.

${ }^{\mathrm{b}}$ Semianalytical model $(\mathrm{SR}+\mathrm{BS})$.

The results of our calculations of the energy spread and the bunch length at the energies $45.5,80$, and $120 \mathrm{GeV}$ for CEPC and FCC-ee are summarized in Tables I and II, respectively. For comparison, in Table I we give the data provided for CEPC [21], while in Table II the results for FCC-ee calculated by Shatilov using the known LIFETRAC code [22,23] are shown.

Here and below, the model data for the $45.5 \mathrm{GeV}$ CEPC were calculated for two variants of the magnetic structure, $Z(2 T)$ and $Z(3 T)$. Because of the closeness of the results, they are averaged.

The reported numerical and analytical results are in satisfactory agreement. However, these values do not take into account the bunch lengthening due to the beam coupling impedance.

\section{COMBINED MODEL WITH BUNCH LENGTHENING}

Since most of the bunch lengthening in both CEPC [24] and FCC-ee [25] is due to the potential well distortion below the microwave instability threshold, at each iteration step we can include the impedance-related bunch lengthening by calculating the synchrotron frequency variation in (6) due to the imaginary part of the impedance [26]:

$$
\nu_{s}^{2}=\nu_{s 0}^{2}\left(1-\frac{\xi}{2 \pi} Z_{\mathrm{pot}}\right)
$$

where [26]

$$
Z_{\mathrm{pot}}=\sum_{p=-\infty}^{\infty} \operatorname{Im} Z\left(p \omega_{0}\right) \frac{J_{1}\left(p \omega_{0} \widehat{\tau}\right)}{\omega_{0} \widehat{\tau} / 2} \widehat{\lambda}\left(p \omega_{0}\right),
$$


with $\hat{\lambda}$ the Fourier transform of the bunch line density, $\hat{\tau}$ the amplitude of synchrotron oscillations, and $J_{1}$ the Bessel function.

If, for simplicity, we assume that the total bunch lengthening is determined by pure inductive impedance, then the following self-consistent solution can be found. For a Gaussian bunch,

$$
Z_{\mathrm{pot}}=\frac{\sqrt{2 \pi}}{\left(\omega_{0} \sigma\right)^{3}} \cdot Z_{c}
$$

where $\sigma=\sigma_{z} / c$. The quantity $Z_{c}$ does not depend on bunch lengthening, i.e., remains constant, and can be determined from the equation describing the lengthening effect in the case of no beam collision [26]:

$$
x_{0}^{3}-x_{0}-\frac{\xi}{2 \pi} Z_{c} \cdot \frac{\sqrt{2 \pi}}{\left(\omega_{0} \sigma_{S R}\right)^{3}}=0
$$

with $x_{0}=\sigma_{0} / \sigma_{S R}$, any known ratio of the increased beam length $\sigma_{z 0}=c \sigma_{0}$ to the length $\sigma_{z, S R}=c \sigma_{S R}$ determined by radiation in bending magnets (SR). The $Z_{c}$ constant is defined by the equation

$$
Z_{c}=\frac{\sqrt{2 \pi}}{\xi}\left(x_{0}^{3}-x_{0}\right)\left(\omega_{0} \sigma_{S R}\right)^{3},
$$

where parameter $\xi$ depends on the bunch current $I_{b}$ :

$$
\xi=\alpha_{p} e I_{b} / \nu_{s}^{2} E
$$

Below the microwave threshold, the inductive impedance decreases the synchrotron tune and, accordingly, increases the bunch length without affecting the energy spread. At the same time, the BS seeks to increase the energy spread and thereby lengthens the bunch, depending on the synchrotron tune variation. Under equilibrium conditions, there must be a self-consistent solution for all three parameters of that interplay. Let $x=\sigma / \sigma_{S R}$ be a steady-state lengthening ratio in such conditions. Considering (8) and (10), Eq. (7) can be rewritten in the form

$$
\nu_{s}^{2}=\nu_{s 0}^{2}\left(1-\frac{x_{0}^{3}-x_{0}}{x^{3}}\right)
$$

Given the contribution of BS to the diffusion coefficient, we have from Eq. (6)

$$
x^{2}=\frac{D_{B S}+D_{S R}}{D_{S R}} \cdot \frac{\nu_{s 0}^{2}}{\nu_{S}^{2}}=\eta \frac{\nu_{S 0}^{2}}{\nu_{S}^{2}} .
$$

Combining Eqs. (12) and (13) gives an equation for a self-consistent solution for the bunch lengthening:

$$
\frac{x^{3}-\eta x}{x_{0}^{3}-x_{0}}=1
$$

It is easy to see that at $\eta=1$ (no BS) Eq. (14) takes a well-known form, describing the lengthening versus bunch current below the turbulence threshold $\left(x_{0}^{3}-x_{0} \propto I_{b}\right)$.

In principle, an iterative algorithm to solve the problem in the described approach includes the following steps: (i) At the first step, set $x=x_{0}$ using the available data on bunch lengthening in CEPC and FCC-ee obtained at the nominal bunch current as a result of only PWD, i.e., without taking into account BS. (ii) From Eq. (1), calculate the BS loss with a current value of the bunch length $\sigma_{z}^{(i)}=x^{(i)} \sigma_{z, S R}$. (iii) Using Eqs. (3)-(5), find the corresponding energy spread $\sigma_{E}^{(i)}$ in the presence of BS. (iv) Recalculate the synchrotron tune from Eq. (12). (v) Calculate a new value of the bunch length $\sigma_{z}^{(i+1)}$ from Eq. (6). (vi) Based on a comparison of $\sigma_{z}^{(i)}$ and $\sigma_{z}^{(i+1)}$, either repeat the cycle from point (ii) or end the iteration.

A step-by-step search of a solution takes quite a long time. Therefore, by setting the initial length in the suspected region of existence of the solution, the calculation time can be reduced to several minutes.

The described algorithm corresponds to a situation when the beams at the nominal currents are brought into collision. An alternative is the sequential accumulation of currents to nominal values in colliding beams (the bootstrapping mode). The algorithm will be complicated by the need to recalculate the magnitude of the impedance at each step of the current increment.

\section{NUMERICAL SIMULATION}

In order to check the model predictions, we use the numerical code IBB [21]. This code performs self-consistent 3D numerical simulations including beamstrahlung and the longitudinal beam coupling impedance. The simulations have been carried out for the stable tune areas free of the recently discovered $X-Z$ coherent beam-beam instability [27].

Figure 2 shows an example of such simulations for FCCee at $45.6 \mathrm{GeV}$. The upper plot demonstrates the energy spread evolution versus the number of revolutions turns, while the bottom one shows the bunch length convergence. For the FCC-ee parameters, the beamstrahlung has the dominating role in the beam dynamics. However, in Fig. 2, we still can see the effect of interplay of the two effects. 

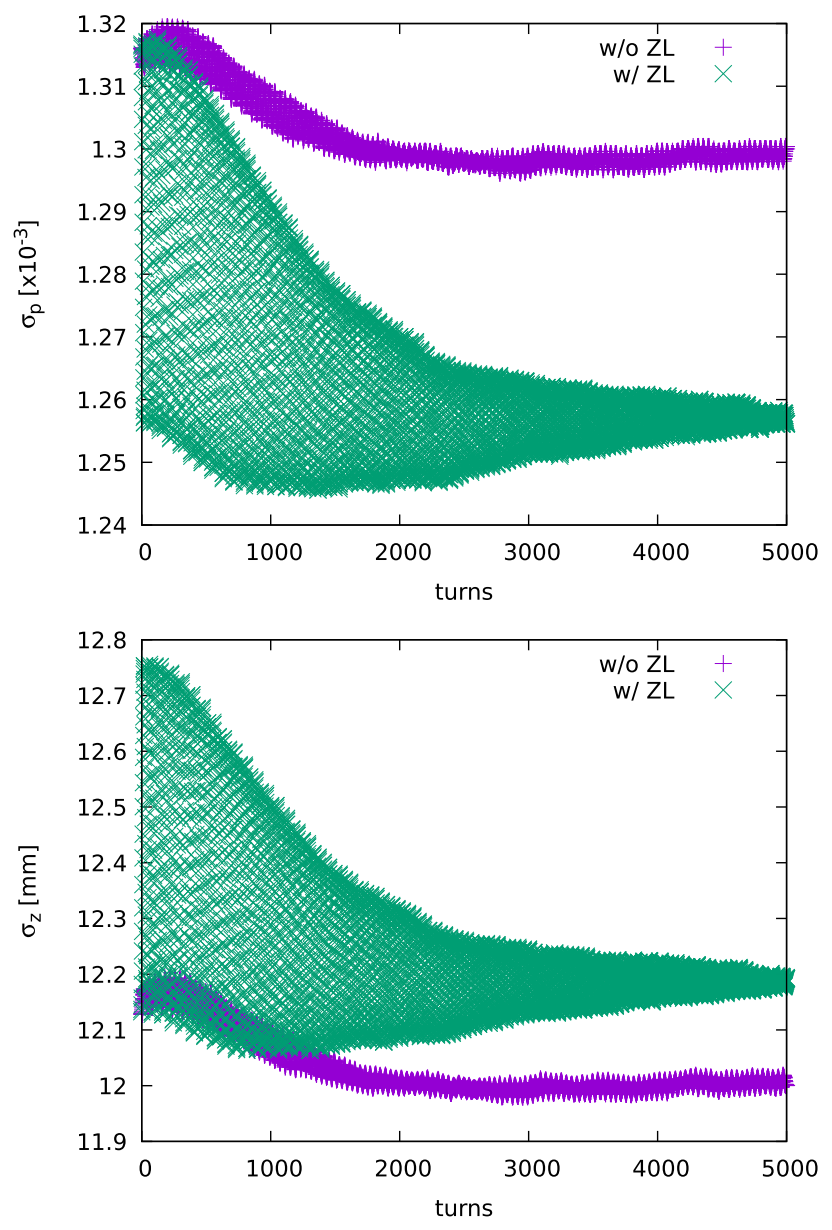

FIG. 2. The energy spread (top) and the bunch length (bottom) versus number of revolution turns with (green traces) and without (viola curves) beam coupling impedance at the 45.6 GeV FCC-ee.

As expected, adding the longitudinal impedance, the bunch becomes somewhat longer and the energy spread slightly reduces.

\section{COMPARISON OF RESULTS}

Our calculations taking into account the combined effect of both BS and PWD effects were performed for CEPC at energies of 45.5, 80, and $120 \mathrm{GeV}$ (see Table III) with the data on lengthening due to PWD [2]. For instance, at $45.5 \mathrm{GeV}$, the latter is from 2.42 to $5.1 \mathrm{~mm}$.

For FCC-ee, due to the lack of similar data for 80 and $120 \mathrm{GeV}$, the calculations were carried out only for 45.6 GeV (see Table IV). The lengthening due to PWD is from 3.5 to $6 \mathrm{~mm}$ [6]. For comparison, both tables (Tables III and IV) show also the results of the simulation [21] accounting for beam lengthening due to the coupling impedance. In the tables, the beam length and energy spread are given taking into account the already complete energy losses on SR and BS. As can be seen from the comparison, even despite such a simplification used, the
TABLE III. The CEPC beam energy spread and length as well as the synchrotron tune in arbitrary units due to the combined effect of SR, BS, and PWD.

\begin{tabular}{lccc}
\hline \hline$E[\mathrm{GeV}]$ & 45.5 & 80 & 120 \\
$\sigma_{E}$ & $0.00105^{\mathrm{a}}$ & $0.00126^{\mathrm{a}}$ & $0.00137^{\mathrm{a}}$ \\
& $0.0010664^{\mathrm{b}}$ & $0.00118^{\mathrm{b}}$ & $0.00132^{\mathrm{b}}$ \\
$\sigma_{z}[\mathrm{~mm}]$ & $7.35^{\mathrm{a}}$ & $5.89^{\mathrm{a}}$ & $4.1^{\mathrm{a}}$ \\
& $7.65^{\mathrm{b}}$ & $5.8^{\mathrm{b}}$ & $3.975^{\mathrm{b}}$ \\
$\nu_{s} / \nu_{s 0}$ & $0.859^{\mathrm{b}}$ & $0.868^{\mathrm{b}}$ & $0.904^{\mathrm{b}}$ \\
\hline \hline
\end{tabular}

${ }^{\mathrm{a}}$ Beam-beam simulation [21].

${ }^{\mathrm{b}}$ Semianalytical model (SR + BS + PWD).

TABLE IV. The FCC-ee beam energy spread and length as well as the synchrotron tune parameter due to the combined effect of SR, BS, and PWD.

\begin{tabular}{lc}
\hline \hline$E[\mathrm{GeV}]$ & 45.6 \\
$\sigma_{E}$ & $0.00126^{\mathrm{a}}$ \\
& $0.00132^{\mathrm{b}}$ \\
$\sigma_{z}[\mathrm{~mm}]$ & $12.2^{\mathrm{a}}$ \\
& $12.6^{\mathrm{b}}$ \\
$\nu_{s} / \nu_{s 0}$ & $0.964^{\mathrm{b}}$ \\
\hline \hline
\end{tabular}

${ }^{\mathrm{a}}$ Beam-beam simulation [21].

${ }^{\mathrm{b}}$ Semianalytical model (SR + BS + PWD).

results of semianalytical calculations are in reasonable agreement with numerical modeling.

\section{CONCLUSIONS}

Using an approximate method that does not require the use of dedicated beam-beam simulation codes, a selfconsistent stationary solution of the energy diffusion equation with BS is found.

In addition, an algorithm has been developed for calculating the beamstrahlung effect taking into account bunch lengthening caused by the potential well distortion due to beam coupling impedance.

The proposed semianalytical model has been used to calculate the equilibrium values of the bunch length and energy spread as well as the synchrotron frequency reduction for the CEPC and FCC-ee colliders at their various target energies.

A comparison of the data calculated by using the model with the available data obtained previously with the beambeam simulation codes has been made. The differences in the beam length (and energy spread), taking into account only BS, are $1.5 \%$ for CEPC and about $2 \%$ for FCC-ee. Given the combined effects of BS and PWD effects, these differences do not exceed 3\% for CEPC and 3.5\% for FCCee, respectively.

Thus, the proposed method allows estimating accurately the BS effect, alone and in combination with PWD, on the longitudinal beam size, energy spread, and synchrotron 
frequency reduction without resorting to a full-scale beambeam simulation.

\section{ACKNOWLEDGMENTS}

The authors thank Dmitry Shatilov for useful discussions.

[1] A. Abada, M. Abbrescia, SS. AbdusSalam, I. Abdyukhanov, J. A. Fernandez, A. Abramov, M. Aburaia, A. O. Acar, P. R. Adzic, P. Agrawal et al., FCC-ee: The lepton collider: future circular collider conceptual design report volume 2, Eur. Phys. J. Special Topics 228, 261 (2019).

[2] CEPC Study Group, CEPC conceptual design report: Volume 1-Accelerator, arXiv:1809.00285.

[3] P. Raimondi, D. Shatilov, and M. Zobov, Beam-beam issues for colliding schemes with large Piwinski angle and crabbed waist, Report No. LNF-07-003-IR, arXiv:physics/ 0702033.

[4] M. Zobov et al., Test of Crab-Waist Collisions at DAФNE Phi Factory, Phys. Rev. Lett. 104, 174801 (2010).

[5] V. Telnov, Restriction on the Energy and Luminosity of e+e- Storage Rings due to Beamstrahlung, Phys. Rev. Lett. 110, 114801 (2013).

[6] E. Belli, M. Migliorati, and M. Zobov, Impact of the resistive wall impedance on beam dynamics in the Future Circular $e^{+} e^{-}$Collider, Phys. Rev. Accel. Beams 21, 041001 (2018).

[7] Y. Zhang, C. T. Lin, N. Wang, and C. H. Yu, Crosstalk of Beam-Beam Effect and Longitudinal Impedance at CEPC, in Proceedings of the 10th International Particle Accelerator Conference (IPAC'19), Melbourne, Australia (JACoW, Geneva, 2019), pp. 247-249, https://doi.org/ 10.18429/JACoW-IPAC2019-MOPGW068.

[8] D. Leshenok, S. Nikitin, and M. Zobov, Model to study interplay between beamstrahlung and coupling impedance in future lepton colliders, arXiv:2004.02553.

[9] KEK press release, Superkekb collider achieves the world's highest luminosity, https://www.kek.jp/en/newsroom/ 2020/06/26/1400/.

[10] A. E. Bondar, V. V. Anashin, V. M. Aulchenko, E. M. Baldin, A. K. Barladyan, A. Yu. Barnyakov, I. Yu. Basok, O. L. Beloborodova, A. E. Blinov, V.E. Blinov et al., Project of a Super Charm-Tau factory at the Budker Institute of Nuclear Physics, Phys. At. Nucl. 76, 1072 (2013).

[11] Q. Luo, W. Gao, J. Lan, W. Li, and D. Xu, Progress of conceptual study for the accelerators of a 2-7 GeV Super Tau Charm facility at China, in Proceedings of the 10th International Particle Accelerator Conference (IPAC'19), Melbourne, Australia (JACoW, Geneva, 2019), pp. 643-645.

[12] See, for example, A. Piwinski, Synchrotron resonances, in Handbook of Accelerator Physics, and Engineering, edited by A. W. Chao, K. H. Mess, M. Tigner, and F. Zimmermann (World Scientific, Singapore, 2013), p. 96.

[13] G. E. Fischer, A brief note on the effect of bunch length on the luminosity of a storage ring with low beta at the interaction point, SPEAR Note No. 154, 1972.

[14] P. Raimondi, M. Zobov, and D. Shatilov, Suppression of beam-beam resonances in crab waist collisions, in Conf. Proc. C 0806233, WEPP045 (2008).

[15] E. Levichev and S. Nikitin, Concept of waveguide Compton monitor of beam energy in high energy e+e-collider, J. Instrum. 11, P06005 (2016).

[16] S. Nikitin, Formulae for estimating average particle energy loss due to beamstrahlung in supercolliders, arXiv:2002 $.01173 v 1$.

[17] K. Takayama, Potential of a 3-dimensional halo charge distribution, IEEE Trans. Nucl. Sci. 30, 2661 (1983).

[18] D. Leshenok and S. Nikitin, Effect of energy loss on accuracy of precision energy measurements in CEPC, in Proceedings of the 2019 International Workshop on the High Energy Circular Electron Positron Collider, 18-20 November 2019, Beijing, China, https://indico.ihep.ac .cn/event/9960/session/8/contribution/195/material/slides/0 .pptx.

[19] M. Sands, The physics of electron storage rings: an introduction, Report No. SLAC-R-121, 1970.

[20] V. Telnov, New features of beamstruhlung important for crab-waist $e^{+} e^{-}$colliders, in Proceedings of IPAC'2018, WEYGBE3 (2018), http://accelconf.web.cern.ch/ipac2018/ talks/weygbe3_talk.pdf.

[21] IBB code, 2020.

[22] D. Shatilov, Beam-beam simulations at large amplitudes and lifetime determination, Part. Accel. 52, 65 (1996), https://inspirehep.net/literature/431185.

[23] D. Shatilov, FCC-ee parameter optimization, ICFA Beam Dyn. Newslett. 72, 30 (2017), https://inspirehep .net/literature/1645012.

[24] N. Wang, Y. Zhang, Y. Liu, S. Tian, K. Ohmi, and C. Li, Mitigation of beam instabilities in CEPC, accepted for publication in Proceedings of the ICFA mini-Workshop on MBCI, Zermatt, Switzerland, 2019.

[25] E. Belli, P. C. Pinto, G. Rumolo, A. Sapountzis, T. Sinkovits, M. Taborelli, B. Spataro, M. Zobov, G. Castorina, and M. Migliorati, Electron cloud buildup and impedance effects on beam dynamics in the future circular $e+e-$ collider and experimental characterization of thin $\mathrm{TiZrV}$ vacuum chamber coatings, Phys. Rev. Accel. Beams 21, 111002 (2018).

[26] Handbook of Accelerator Physics, and Engineering edited by A. W. Chao, K. H. Mess, M. Tigner, and F. Zimmermann (World Scientific, Singapore, 2013).

[27] K. Ohmi, N. Kuroo, K. Oide, D. Zhou, and F. Zimmermann, Coherent Beam-Beam Instability in Collisions with a Large Crossing Angle, Phys. Rev. Lett. 119, 134801 (2017). 